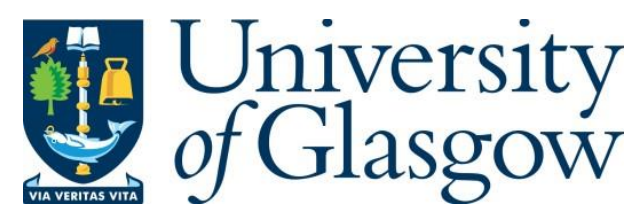

Rizwan, M., Adhur Kuttty, A., Kgwadi, M., Drysdale, T. D., Sydanheimo, L., Ukkonen, L. and Virkki, J. (2017) Possibilities of Fabricating Copper-based RFID Tags with Photonic-sintered Inkjet Printing and Thermal Transfer Printing. IEEE Antennas and Wireless Propagation Letters, 16, pp. 1828-1831.

There may be differences between this version and the published version. You are advised to consult the publisher's version if you wish to cite from it.

http://eprints.gla.ac.uk/147782/

Deposited on: 12 September 2017

Enlighten - Research publications by members of the University of Glasgow http://eprints.gla.ac.uk 


\title{
Possibilities of Fabricating Copper-Based RFID Tags With Photonic-Sintered Inkjet Printing and Thermal Transfer Printing
}

\author{
Muhammad Rizwan, Student Member, IEEE, Ajith Adhur Kutty, Student Member, IEEE, \\ Monageng Kgwadi, Student Member, IEEE, Timothy D. Drysdale, Member, IEEE, \\ Lauri Sydänheimo, Member, IEEE, Leena Ukkonen, Member, IEEE, and Johanna Virkki, Member, IEEE
}

\begin{abstract}
This letter studies the possibilities of manufacturing copper-based passive ultrahigh frequency (UHF) radio frequency identification (RFID) tags using inkjet and thermal printing on two substrate materials, polyimide (Kapton), and a polyester-based substrate (Flexcon THERMLfilm). Both printing methods are tested to fabricate different tag designs, and the performance of successfully printed tags is evaluated using wireless measurements. Measurement results show that both printing methods, while using copper material, can be used to effectively fabricate passive UHF RFID tag antennas on selected substrates.
\end{abstract}

Index Terms-Copper, inkjet printing, Kapton, photonic sintering, radio frequency identification (RFID), THERMLfilm, thermal printing.

\section{INTRODUCTION}

A DDITIVE manufacturing is a promising paradigm for the fabrication of antennas for pervasive Internet of Things applications, as it has the capacity to integrate versatile materials. Antennas are the critical enabling components of the future wireless world, and material choices in antenna fabrication will have huge impact on the environment, as well as on the cost of the fabricated wireless devices.

Inkjet [1] and thermal [2] printing are two attractive additive manufacturing methods for printing antennas, as shown in our previous work [3]-[5]. Although these printing methods offer new opportunities for antenna manufacturing, the cost of printed conductor materials needs to be reduced as well. Using copper inks, with potentially lower material costs compared to

This work was supported in part by the European Commission from the Seventh Framework Programme through AdvIOT project (www.adviot.eu), in part by the Academy of Finland, and in part by the TEKES.

M. Rizwan, A. Adhur Kutty, L. Sydänheimo, L. Ukkonen, and J. Virkki are with the Department of Electronics and Communications Engineering, Tampere University of Technology, Tampere 33720, Finland (e-mail: muhammad. rizwan@tut.fi; ajith.adhurkutty@tut.fi; lauri.sydanheimo@tut.fi; leena. ukkonen@tut.fi; johanna.virkki@tut.fi).

M. Kgwadi is with the Electronics and Nanoscale Engineering Research Division, University of Glasgow, Glasgow G12 8QQ, U.K. (e-mail: m.kgwadi.1@research.gla.ac.uk).

T. D. Drysdale is with the Department of Engineering and Innovation, Open University, Milton Keynes MK7 6AA, U.K. (e-mail: tim.drysdale@ open.ac.uk).

Color versions of one or more of the figures in this letter are available online at http://ieeexplore.iee.org

Digital Object Identifie silver inks, for printing electronics has been an active research area during the recent years [6]-[8]. In [8], screen- and inkjetprinted copper conductors were laser-cured on four different substrate materials, i.e., flexible Kapton (polyimide), polyethylene terephthalate (PET), paper, and polyester/cotton fabric. The main focus of that work was laser curing. As an alternative, photonic curing/sintering can be used for inkjet-printed conductors, which is a topic explored in this work.

In our previous studies, we compared thermal-printed aluminium and inkjet-printed silver [3], along with the reliability study of radio frequency identification (RFID) tags printed with both additive manufacturing methods [4]. The next step was the detailed performance analysis of inkjet- and thermal-printed passive ultrahigh-frequency (UHF) RFID tags, and also the possibility of printing copper with thermal transfer print [5]. In all our previous works, heat sintering has been the only studied sintering method.

Until now, little work has been done to demonstrate the true potential of copper-based materials in RFID tag antenna fabrication. The novelty of this work is: 1) studying the possibilities of printing copper-based antennas on a polyimide substrate (Kapton HN, referred to as Kapton or KP) and on a novel polyesterbased substrate material (Flexcon THERMLfilm Select 21944E (TC-390), referred to as THERMLfilm or TF) using inkjet and thermal printing; 2) studying the photonic sintering for inkjetprinted tag antennas on THERMLfilm. Adhesion is one of the key factors in good printing in our study; we have also tried inkjet printing with and without oxygen plasma treatment to check if it improves the adhesion problem of THERMLfilm for better printing and photonic sintering. The main emphasis of the results is on the wireless read range measurements and radiation characteristics of the successfully printed RFID tags. The letter ends with concluding remarks and future work.

\section{MODEling AND MANUfACTURING OF TAGS}

\section{A. Tag Geometry and Modeling}

Three different tag antenna designs were selected for this study, which helps excluding the impact of different antenna geometries on the results. ANSYS HFSS v15 was used for the modeling and simulation of these tags. Kapton and THERMLfilm were modeled as 50- and 70- $\mu$ m-thick substrates with relative dielectric constants of 3.2 and 3.0, respectively. The simulation model used the thickness and conductivity of 


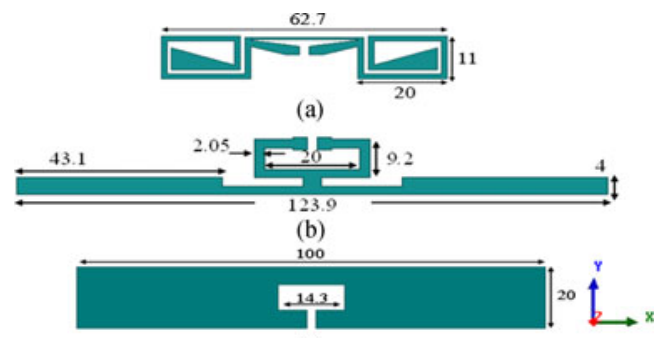

(c)

Fig. 1. Tag antennas with dimensions in millimeters. (a) Tag 1. (b) Tag 2. (c) Tag 3 .
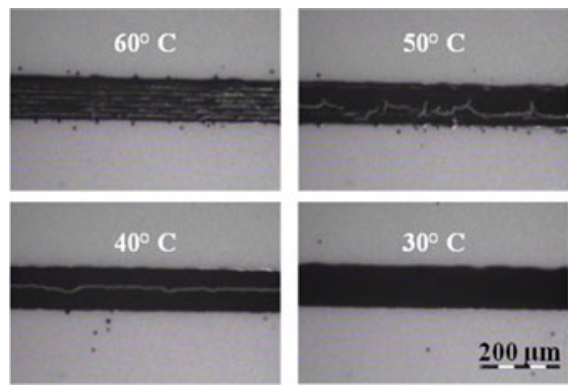

Fig. 2. Effect of platen temperature on printed pattern.

the conductor according to the measured values. Fig. 1 shows the studied tag antenna geometries.

\section{B. Tag Manufacturing}

1) Inkjet Printing: Inkjet printing has previously been used for manufacturing UHF RFID tags on versatile substrates, such as wood, paper, and cardboard materials [1], [9], but the use of THERMLfilm as a substrate for the inkjet-printed conductor is relatively new [3]-[5]. The inkjet printing in this study was done using a Fujifilm Dimatix DMP-2831 material inkjet printer. The ink used was ANI Cu-IJ70 copper nanoparticle ink [10] (with achievable conductivity range of $\left.1.4 \times 10^{7}-2.0 \times 10^{7} \mathrm{~S} / \mathrm{m}\right)$. The printing parameters that affect the print quality on any substrate are cartridge temperature, platen temperature, jetting voltage, jetting frequency, and the drop spacing or pattern resolution. As presented in [3], the optimum resolution for inkjet printing was obtained using a drop test. During the inkjet printing process on THERMLfilm, a platen temperature of only $30^{\circ} \mathrm{C}$ was used to avoid the quick absorbing and drying of the ink, which produces cracks on the printed layer (see Fig. 2), as the paper starts absorbing the ink quickly, leaving gaps into the printed pattern. In our previous studies [3]-[5], a platen temperature of $60{ }^{\circ} \mathrm{C}$ used for printing silver nanoparticle ink on THERMLfilm caused no cracks into the printed pattern. Other parameters were set to achieve a proper drop formation. Single-layer printing was done. The key parameters for inkjet printing on both substrates are given in Table I.

The copper nanoparticle ink needs photonic sintering to produce a conductive pattern. Before photonic sintering is performed, the printed antennas were preheat sintered at $100{ }^{\circ} \mathrm{C}$ for $30 \mathrm{~min}$ as recommended by the manufacturer. Due to this preheating process, no photonic absorption occurs during photonic sintering. Subsequently, photonic sintering was performed using Xenon Sinteron 2010-L. The two main user-alterable parameters of the sintering machine are pulse voltage $V$ and pulse duration
TABLE I

OPTIMIZED KEY PARAMETERS FOR INKJET PRINTING

\begin{tabular}{lcc}
\hline \hline Parameter & $\mathrm{KP}(\mathrm{Cu})$ & $\mathrm{TF}(\mathrm{Cu})$ \\
\hline Platen Temperature $\left({ }^{\circ} \mathrm{C}\right)$ & 45 & 30 \\
Cartridge Temperature $\left({ }^{\circ} \mathrm{C}\right)$ & 40 & 30 \\
Max. Jetting Frequency $(\mathrm{kHz})$ & 5 & 5 \\
Jetting Voltage $(\mathrm{V})$ & 24 & 25 \\
Pattern Resolution $(\mathrm{DPI})$ & 726 & 726 \\
\hline
\end{tabular}

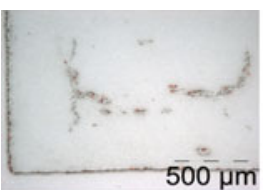

(a)

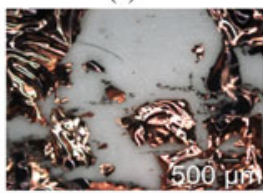

(c)

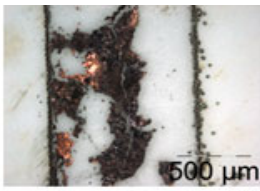

(b)

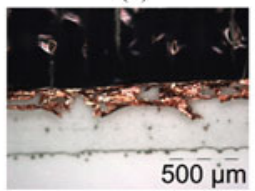

(d)
Fig. 3. Printed pattern after sintering. (a) $2200 \mathrm{~V}, 1700 \mu$ s. (b) $2000 \mathrm{~V}$, $1500 \mu \mathrm{s}$. (c) $2000 \mathrm{~V}, 1300 \mu \mathrm{s}$. (d) $1800 \mathrm{~V}, 1000 \mu \mathrm{s}$.

$t$, which can be related to total energy $E$ supplied during sintering as follows, according to the machine's operating manual:

$$
E=\left(\frac{V}{3120}\right)^{2.4} \times t
$$

where $E$ is the energy in joules per pulse, $V$ is the pulse voltage value in volts, and $t$ is the time in microseconds.

Optimization of the voltage and pulse duration is necessary for each combination of materials [9]. On the Kapton substrate, one flash of $2200 \mathrm{~V}$ with $1700 \mu$ s pulse duration was found to be optimal. On the THERMLfilm substrate, the optimized sintering conditions used with Kapton resulted in the printed ink being partially removed from the substrate. This shows that the energy is too high for the printed pattern. Different combinations of number of flashes and pulse duration, as shown in Fig. 3, were used to sinter, but either the ink was removed from the surface or burnt due to the high energy, or it stayed nonconductive. The reason for nonconductive pattern is that the energy transferred during the sintering is not enough for the pattern to be conductive. However, increasing the energy more does not help the pattern to get conductive, but due to the low adhesion of the ink on THERMLfilm, all the conductor gets removed. As a test, multiple layers of copper (two to six layers) were printed on THERMLfilm, but it gave the same results after sintering. Despite our systematic study, we were not able to find any optimum sintering conditions to have a conductive pattern on THERMLfilm.

To improve the adhesion, oxygen plasma treatment was applied on the THERMLfilm substrate, using Diener Pico, equipped with optional reactive-ion etching style electrodes. The parameters used were $30 \mathrm{~W}$ power, 0.3 mbar pressure, 2 min time, and pure oxygen gas. These parameters are set to have the best treatment for THERMLfilm. After the treatment, the printing and sintering processes were repeated, but still no improvement was observed after the sintering process, and the pattern remained nonconductive or removed from substrate. 


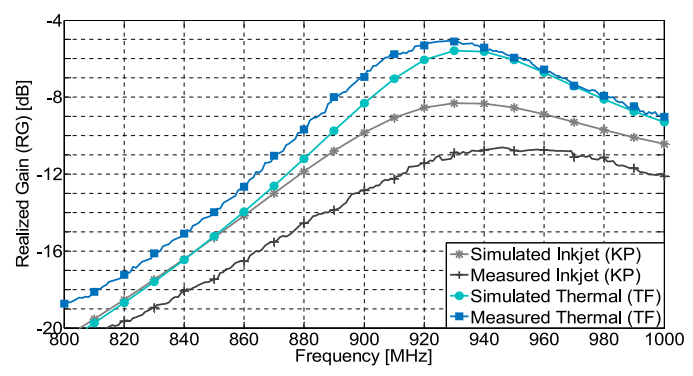

(a)

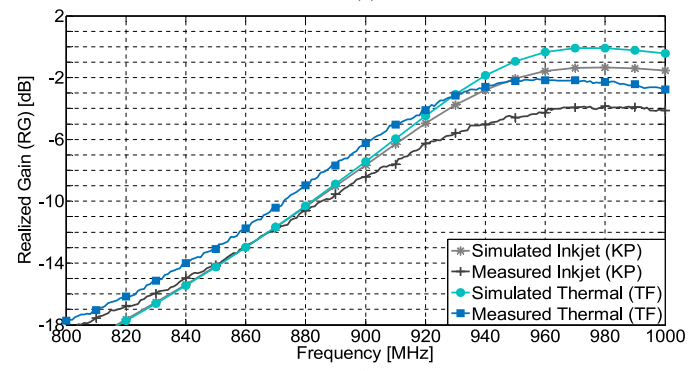

(b)

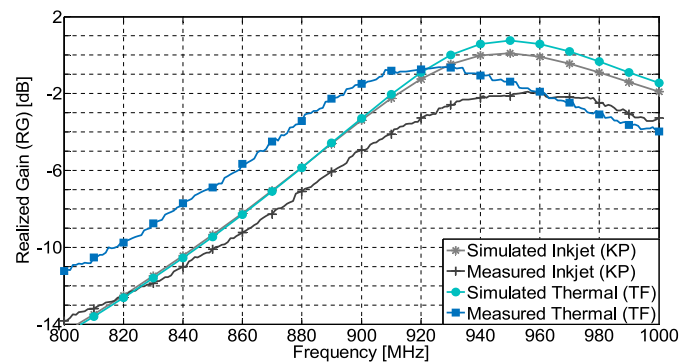

(c)

Fig. 4. Simulated and measured realized gains. (a) Tag 1. (b) Tag 2. (c) Tag 3.

This shows that with THERMLfilm, the photonic sintering process will not be suitable if copper nanoparticle ink is used.

2) Thermal Printing: Thermal printing in this study was done using a Zebra S4M 300-dots/inch (dpi) thermal printer at a speed setting of $5 \mathrm{~cm} / \mathrm{s}$ and darkness 30 . Thermal printing works on the principle of thermal transfer print where a thermal print head is used to selectively heat a conductive ribbon, which then adheres to the substrate [5]. It is a quick method, and tags require no further processing after the printing, compared to inkjet printing, which requires the sintering process. For this work, the ribbon employed was IIMAK Metallograph Conductive Thermal Transfer Ribbon, which features 340-nm-thick copper and $1-\mu \mathrm{m}$-thick heat-sensitive acrylic adhesive. Thermal printing worked well on THERMLfilm, but was not successful on Kapton. The main reason for the failure is the poor adhesiveness of the thermal ribbon on Kapton. The oxygen plasma treatment cannot be applied to thermal printing because the substrate is rolled inside the machine and this removes the effects of treatment before the printing can be done.

\section{MEASUREMENT RESULTS AND DisCUSSION}

\section{A. Sheet Resistance and Conductivity}

The thicknesses of the inkjet-printed and thermal-printed copper conductors were measured to be 0.45 and $0.34 \mu \mathrm{m}$, respectively, using a Veeco Wyko NT1100 optical profiling system. In both of the printing methods, only single printing pass was done.

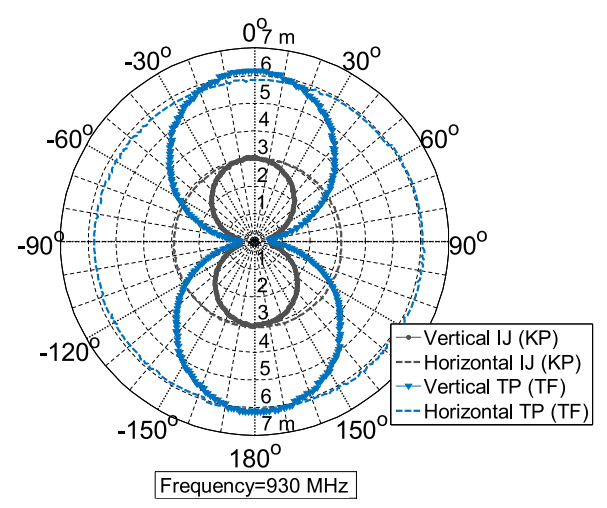

(a)

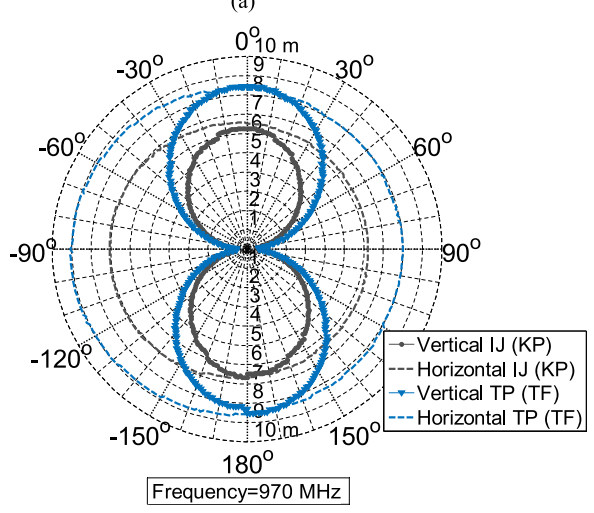

(b)

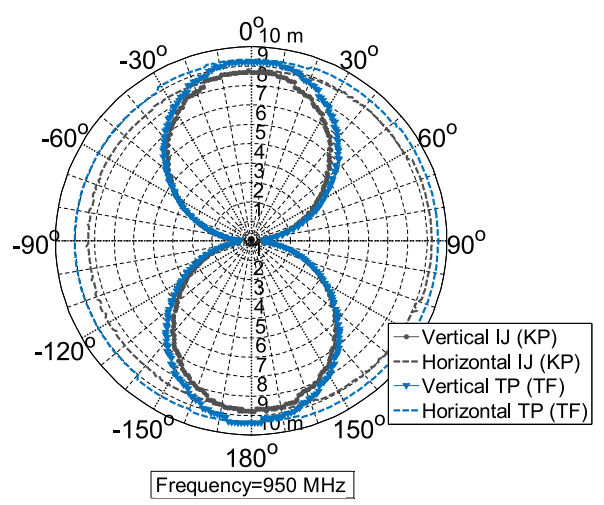

(c)

Fig. 5. Measured read ranges of printed Tags. (a) Tag 1. (b) Tag 2. (c) Tag 3.

A four-probe measurement using Greek cross structures was used to measure the sheet resistances [11]. The measured sheet resistance $\left(R_{\mathrm{S}}\right)$ and thickness $(t)$ were then used to calculate the resistivity $(\rho)$ and conductivity using the following relation:

$$
\rho=R_{\mathrm{s}} \times t .
$$

The conductivity of the inkjet-printed copper on Kapton was found to be around $1.50 \times 10^{7} \mathrm{~S} / \mathrm{m}$, while the thermal-printed copper on THERMLfilm showed a higher conductivity, around $3.9 \times 10^{7} \mathrm{~S} / \mathrm{m}$. The measured sheet resistance of the inkjetprinted copper on Kapton $(148 \mathrm{~m} \Omega$ ) is lower than previously reported for the same ink on Kapton [9].

\section{B. Realized Gain and Wireless Read Range}

NXP UCODE G2iL RFID integrated circuits (ICs) were attached to the antenna terminals after printing using a silver epoxy (Circuit Works CW2400). 
TABLE II

COMPARISON OF PRINTEd TAGS IN THIS ANd PREVIOUS StUdies

\begin{tabular}{lccccc}
\hline \hline Ref. & Method, Substrate & Ink & Tag 1 & Tag 2 & Tag 3 \\
\hline [3], [5] & Thermal transfer print (Kapton) & $\mathrm{Al}$ & Fabrication not successful \\
[5], This work & & $\mathrm{Cu}$ & & & \\
[3]-[5] & Thermal transfer print (THERMLfilm) & $\mathrm{Al}$ & $4.4 \mathrm{~m}$ & $7.7 \mathrm{~m}$ & $9.8 \mathrm{~m}$ \\
[5], This work & & $\mathrm{Cu}$ & $6.6 \mathrm{~m}$ & $9.0 \mathrm{~m}$ & $10.6 \mathrm{~m}$ \\
[3], [5] & Inkjet print (Kapton) & $\mathrm{Ag}$ & $5.8 \mathrm{~m}$ & $8.6 \mathrm{~m}$ & $10.6 \mathrm{~m}$ \\
This work & & $\mathrm{Cu}$ & $3.4 \mathrm{~m}$ & $7.1 \mathrm{~m}$ & $10.8 \mathrm{~m}$ \\
[3]-[5] & Inkjet print (THERMLfilm) & $\mathrm{Ag}$ & $4.2 \mathrm{~m}$ & $7.9 \mathrm{~m}$ & $9.2 \mathrm{~m}$ \\
This work & & $\mathrm{Cu}$ & Fabrication not successful \\
\hline \hline
\end{tabular}

The wireless performance of the tags was evaluated using a Voyantic Tagformance RFID measurement unit [3]. The simulated and measured realized gains versus frequency of the tags are given in Fig. 4. The realized gain is the product of gain and impedance mismatch factor. The peaks correspond to the frequency of best matching as well as the peak read range. The read ranges can be computed using the realized gains as

$$
R_{\text {range }}=\left(\frac{\lambda}{4 \pi}\right) \sqrt{\frac{G_{t} \times \mathrm{EIRP}}{\mathrm{P}_{\text {chip }}}}
$$

where $G_{\mathrm{t}}$ is the realized gain of the tag, $\lambda$ is the wavelength, $P_{\text {chip }}$ is the minimum power required to activate the chip, and EIRP is the effective isotropically radiated power, which is limited to $3.28 \mathrm{~W}$, according to the European regulations.

Results show that the thermal-printed tags show higher realized gains than the inkjet-printed ones. A little shift in the peak realized gain with frequency is observed, compared to the simulation results, for the thermal-printed tags. This shift of the resonance on THERMLfilm can be attributed to the presence of the glassine release cover, which is there to preserve the adhesive at the back of the THERMLfilm. This layer was not included in the calculation of the effective dielectric constant. In practice, when the antennas are deployed, this layer is supposed to be peeled off.

Based on the realized gain results, in the case of Tag 1, the thermal printing shows a measured peak read range of $6.52 \mathrm{~m}$ at $926 \mathrm{MHz}$, and the inkjet-printed tag shows a peak read range of $3.37 \mathrm{~m}$ at $945 \mathrm{MHz}$. The higher conductivity of the thermalprinted copper resulted in a much longer read range compared to the inkjet-printed tags.

For Tag 2, the thermal printing shows a peak read range of $8.91 \mathrm{~m}$ at $957 \mathrm{MHz}$, and the inkjet-printed tag shows a peak read range of $7.12 \mathrm{~m}$ at $970 \mathrm{MHz}$. For Tag 3, the thermal printing shows a measured peak read range of $10.83 \mathrm{~m}$ at $920 \mathrm{MHz}$, and the inkjet-printed tag shows a peak read range of $9.13 \mathrm{~m}$ at $956 \mathrm{MHz}$.

The read ranges in vertical (yz-plane) and horizontal (xy-plane) planes were measured for all the tags in free space, at the frequencies of interest, and are shown in Fig. 5. The frequencies of interest for Tags 1, 2, and 3 are 930, 970, and $950 \mathrm{MHz}$, respectively. For the read range measurements, $1^{\circ}$ variation in angle and 0.1-dB variation in power were used. As can be seen, the read ranges vary in correspondence with the dipole nature of the tags.

Table II summarizes the measured theoretical read ranges of all the printed tags in this and previous studies [3-5]. It can be seen that in our previous study with silver and aluminium materials [3], the measured read ranges of Tag 1 and Tag 2 were around $4-5$ and $8-9 \mathrm{~m}$, respectively. The measured read ranges of silver and aluminium printed tags were slightly lower (1-2 $\mathrm{m})$ than the thermal-printed copper tags, but better (1-2 m) than the inkjet-printed copper tags. This increase in read range is due to the higher conductivity of the thermal-printed copper when compared to earlier printed silver and aluminium conductors.

\section{CONCLUSION}

In this letter, we presented the possibilities of fabricating copper-based passive UHF RFID tags using inkjet and thermal printing on polyimide and on a polyester-based substrate. Three tag antenna geometries were utilized in the study. RFID tag antennas were fabricated successfully by inkjet printing of copper nanoparticle ink on a Kapton substrate and by thermal printing of copper material on a THERMLfilm substrate. The measurement results showed a peak read range of 7-10 $\mathrm{m}$ for the thermal-printed tags on THERMLfilm, due to their excellent conductivity. The inkjet-printed tags on Kapton showed a better sheet resistance than previously reported and had a $2-3 \mathrm{~m}$ shorter read range than the thermal-printed tags on THERMLfilm.

This study shows that inkjet-printed copper has adhesion problems on THERMfilm, and thermal-printed copper on Kapton. However, this can be considered a good opportunity for further research. More future research topics can include studying the reliability of the printed tags, e.g., in high-humidity conditions and after cyclic bending.

\section{ACKNOWLEDGMENT}

The authors would like to thank D. Harrison from IIMAK for donation of copper Metallograph ribbons.

\section{REFERENCES}

[1] L. Yang, A. Rida, R. Vyas, and M. M. Tentzeris, "RFID tag and RF structures on paper substrates using inkjet-printing technology," IEEE Trans. Microw. Theory Techn., vol. 55, no. 12, pp. 2894-2901, Dec. 2007.

[2] M. Kgwadi and T. D. Drysdale, "Diode-switched thermal-transfer printed antenna on flexible substrate," Electron. Lett., vol. 52, no. 4, pp. 258-260, 2016.

[3] M. Rizwan, A. A. Kutty, M. Kgwadi, T. D. Drysdale, L. Ukkonen, and J. Virkki, "Comparitive study of inkjet and thermal printing for fabrication of passive UHF RFID Tags," in Proc. 10th Eur. Conf. Antennas Propag., 2016, pp. 1-5.

[4] M. Rizwan, A. A. Kutty, M. Kgwadi, T. D. Drysdale, L. Ukkonen, and J. Virkki, "Reliability study of flexible inkjet- and thermal-printed RFID antennas in high humidity conditions," in Proc. 10th Eur. Conf. Antennas Propag., 2016, pp. 1-5.

[5] M. Kgwadi, M. Rizwan, A. A. Kutty, J. Virkki, L. Ukkonen, and T. D. Drysdale, "Performance comparison of inkjet and thermal transfer printed passive UHF RFID Tags," Microw., Antennas Propag., vol. 10, no. 14, pp. 1507-1514, Nov. 2016.

[6] Y. Hu, B. An, C. Niu, W. Lv, and Y. Wu, "Application of nano copper conductive ink for printed electronics," in Proc. 15th Int. Conf. Electron. Packag. Technol., 2014, pp. 1565-1567.

[7] J. Kim, B. Lee, J. Y. Lek, R. I. Made, B. Salam and C. L. Gan, "Characterization of copper conductive ink for low temperature sintering processing on flexible polymer substrate," in Proc. 16th Conf. IEEE Electron. Packag. Technol., 2014, pp. 27-30.

[8] Y. Wei, Y. Li, R. Torah, and J. Tudor, "Laser curing of screen and inkjet printed conductors on flexible substrates," in Proc. Symp. Design Test Integr. Packag. MEMS/MOEMS, 2015, pp. 1-5.

[9] Y. Ren, J. Virkki, L. Sydänheimo, and L. Ukkonen, "Optimisation of manufacturing parameters for inkjet-printed and photonically sintered metallic nanoparticle UHF RFID tags," Electron. Lett., vol. 50, no. 21, pp. 1504 1505,2014

[10] Applied Nanotech Holdings, Inc., "Cu-IJ70 Copper nanoparticle Ink," [Online]. Available: http://www.appliednanotech.net, accessed on Mar. 24, 2017.

[11] S. Enderling et al., "Sheet resistance measurement of non-standard cleanroom materials using suspended Greek cross test structures," IEEE Trans. Semicond. Manuf., vol. 19, no. 1, pp. 2-9, Feb. 2006. 